\title{
O. Mandel'stam, Il programma del pane. Come lievita la poesia, a cura di L. Tosi, Jouvence, Milano 2021, pp. 272.
}

Si dovrebbe immaginare una speciale macchina volante, un aereo che in volo tragga da sé, dal proprio interno una seconda parte e un'altra ancora, tutte egualmente dinamiche. Questa l'immagine utilizzata da Osip Mandel'štam per dare corpo-forma visiva alla materia poetica, nella sua Conversazione su Dante. Si potrebbe forse applicare alla struttura, inconsueta, del volume con cui Lia Tosi ripropone un suo innovativo libro del 2004, arricchito di un'ampia parte, del tutto originale, dedicata al rapporto fra Mandel'štam e lo psicologo Lev Vygotskij al punto d'incontro della loro ricerca sull'origine 'biologica' della parola, della parola poetica in particolare. Un libro costruito secondo un principio di tessitura dritto-rovescio, dove la figura finale sarà ricostruita solo a lettura ultimata. La struttura a cornice del primo libro, composto di quattordici capitoli, si suddivide duplicandosi, riportando la traduzione di alcuni saggi di Mandel'štam sulla parola poetica e sulla sua funzione nella cultura percepita come organismo vivente, Parola e cultura, Sulla natura della parola, Umanesimo e tempo contemporaneo, a loro volta incastonati fra due scritti che ne sono poli e insieme asse portante, cintura e capienza, Frumento umano e Il programma del pane. Essi danno senso al titolo del libro e attendono la chiosa finale: Come lievita la poesia. Segue ogni brano o saggio una prima interpretazione dei maggiori studiosi di Mandel'štam, riportata con probità a mo' di incipit per un commento da parte dell'autrice, che si avvale di altro materiale per intervallare l'esegesi, con poesie, brani di prosa, citazioni da altri poeti e prosatori (soprattutto da Rozanov) che a loro volta ne rinsaldano il significato e aprono a nuovi ventagli di senso. Anche Lia Tosi sa l'arte del cucire, sicché sutura abilmente, chiosando le linee di pensiero che va seguendo, rintracciando, interpolando. Seguono ai saggi singole poesie che puntellano il discorso sul Programma del pane, quindi, a mo' di chiusura, le due Odi tarde, entrambe degli anni Trenta: Ode a Stalin e i Versi sul soldato ignoto, Oratorio in otto parti, scrive Jurij Levin, attorno a cui far convergere le fitte linee di attraversamento dell'intera poetica di Mandel'štam. Anche la veste tipografica segue e assicura questa sorta di carotaggio esegetico, affilando fino al cosiddetto 'corpo minore' l'indagine, quasi carne viva, la poesia che vibra in onde, empiti (poryvy), sussulti, poi si distende, con un andamento, si suggerisce, proprio alla parola di un asmatico, quale era il poeta, che sa la difficoltà del dire: "Amo l'apparizione del tessuto, / quando dopo due oppure tre / $\mathrm{ma}$ anche più respiri ansimati / arriva il respiro a pieno petto" (Ottave, novembre 1933). Insegue, Lia Tosi, immagini, metafore, interrato rizoma, singole parole-segnale che tessono nella poetica di Mandel'štam un disegno coerente e persistente. "Certe metafore per Mandel'štam sono come giuramenti, si rinnovano, si conservano”. Così la formazione 
organica della parola poetica, predeterminata come in natura la "foglia embrionale del nasturzio", eppure imprevedibile e spontanea, viene captata dalla vela tesa di un "udito sensibile", un orecchio interno, addestrato alle sponde dello Stige creativo, in cerca del suono amato. "É la figura interna che emana suono, è l'udito poetico che la percepisce", come nelle poesie fra loro concatenate: "Ho dimenticato la parola che volevo dire..." e le gemellari: "Quando scende alle ombre Psiche -vita". Procede, questo indagare a più piste, verso alcuni nuclei itineranti: il calore, la famiglia, il nutrimento, la potenza dinamica della parola in formazione, paragonabile alla "velocità cosmica dell'esplosione di un baccello di nasturzio" (Viaggio in Armenia). Sviluppo organico che aveva trovato anticipazione nelle immagini del saggio Parola e cultura, come "la parola è carne e pane", "chi alzerà la parola [...] un secondo Giosuè" fino alla sacralità eucaristica. Da qui origina il glossario neotestamentario, il fondaco da cui attingere le iterate metafore del campo di grano, del seminatore e delle sementi, del raccolto. Ma se queste si svolgono tutte con le contigue immagini del vomere, "La poesia è un vomere che rivolta il tempo in modo da far riemergere in superficie i suoi strati profondi”, dei solchi e della 'terra nera' fertile, Il programma del pane guarda al cielo, in parte echeggiando le teorie allora in voga di Gurdžiev, che altrettanto ebbero influenza su poeti come Chlebnikov o Zabolockij, sintetizzate nell'esortazione: "Dicono che causa di rivoluzione sia la fame negli spazi interplanetari. Bisogna spargere frumento nell'etere”.

Si parla di una letteratura calda e intima, indossata "come i pantaloni", 'così vicini e così miei”" scriveva Vasilij Rozanov, esaltando l'elemento domestico, familiare della letteratura che in Mandel'štam si conserva come materia organica vivificatrice attraverso i secoli. La lingua umana, dotata di un calore biologico, quasi si identifica, come nella Glossolalija di Andrej Belyj, col luogo in cui risuona e con l'organo che la dice, come l'ancipite significato della parola stessa, lingua. Così Lia Tosi riesce a seguire lo sviluppo di immagini, parole-lemmi itineranti che dalle prime poesie degli anni Dieci crescono nelle solenni forme architettoniche, 'classiche' degli anni Venti, rinnovando nei versi di Tristia gli stretti legami che saldano la poesia e il nome di Mandel'štam ai suoi numi tutelari: Ovidio e Dante.

Parola colta che emana tepore, evoca il "focolare", perché nutrita di filologia affettiva: "la filologia è famiglia, perché ogni famiglia si tiene su intonazioni, citazioni, virgolette”, esalta il calore umano e si oppone, resiste al rigore dei ghiacci inospitali delle terre oltre Ponto, come al rigor mortis della massa umana indistinta (Versi sul soldato ignoto), maciullata in "pastone arabo, tritato" dalla guerra e dagli ordigni bellici, "corpi volanti a sei braccia”, "ali assire di libellule / vibrazioni di angolose tenebre” (Il vento a noi consolazione portó). Cresce negli anni in Mandel'štam l'orrore per la cupa eredità bellica del primo conflitto mondiale e la funesta preveggenza del prossimo futuro, con nuovi micidiali armamenti per il martirio dei singoli corpi in un 'pastone' (mesivo) indistinto, indifferente, per le avide, sornione, ciniche Potenze mondiali. Colpisce la lettura dei reportages di guerra (citati nelle note ai Versi sulsoldato ignoto) che preconizzano l'oggi dell'Afghanistan, a noi tristemente ben noto, colpisce la sollecitudine del poeta per l'umanità, resa campo di spighe falcidiato dall'orrida macchina bellica. Convergono nell'oratorio sul Soldato immagini evangeliche, patristiche, ortodosse, aboliti i nessi logici, gli otto brevi cicli si inanellano per attrazione musicale, condensando la riflessione di anni, aprendo a una visione cosmica davvero originale. Tra le sorprese di questa apertura nell'Ode a Stalin, spicca la qualità della traduzione, mentre l'autonomia del commento scava la progressione dell'immagine originaria del mietitore, sicché Stalin, colui che "ha spostato l'asse del mondo", "vomere gigante", sarà connesso ad Augusto, tormentatore di Ovidio. Eppure Lia Tosi vi legge un desiderio assurdo e pur autentico di riscatto del Tiranno, la speranza che il calore filiale possa "curare" il padre, riconnettendo così l'ultima parte di questo primo libro alla "cura" che sanerà 
la "Guernica cosmica" della belligeranza, in apertura. Conosciamo meglio Mandel'štam, contemporaneo del suo secolo, nella Mosca del "Moskvovšej” (Moscacuci), pronto all'arruolamento - "Io pollone selvatico impaurito della luce / diverrò soldato semplice di quel paese” (Versi sul soldato ignoto).

Mentre l'ultima parte del libro approfondisce e coglie con sottili pinzette d'orefice il complesso meccanismo della morfogenesi della poesia, la sua "natura materica", "fiamma a due lingue" che negli anni Trenta genera la metafora del tessuto, tkan', e della spoletta, del fuso, veretenó, collegando l'azione di sparizione e ricomparsa di fili, immagini, metafore costanti nel tempo. Vicino alla simultanea ricerca di Lev Vygotskij, espressa in Pensiero e parola (Mysl'i slovo), ma ancora nei taccuini ora riordinati con lavoro certosino, dove più si mostra, anche con esplicite citazioni dal poeta, l'affinità e coincidenza della loro ricerca. Mandel'štam, affascinato dalle spiegazioni scientifiche del giovane biologo Boris Kuzin, con cui stringe amicizia nel soggiorno in Armenia, dalla lettura dei Naturalisti (Viaggio in Armenia) suggellata dalla poesia a Lamarck, attinge ora a una nuova terminologia o meglio una affinità di percezione per definire la morfogenesi del linguaggio. Psicologo e poeta individuano concordi, nel 'linguaggio per sé, interiore, insonoro, l'assenza di sintassi ipotattica, il logico costrutto, verticale, del 'linguaggio per gli altri', e la presenza, percepita e indimostrabile, di un magmatico, disarticolato e orizzontale generarsi di suono-immagine, ciò che sta 'prima' del pensiero, parola che si vuole dire.

Questo libro infine, commovente per la sua verità, è scritto in una lingua non convenzionale, robusta, adeguata e inventiva insieme, dove senti risuonare la colta origine toscana, un lessico dantesco a volte, reso però domestico, alla Rozanov, non artefatto, simpatetico e libero. Ed è la lingua con cui Lia Tosi si applica al tradurre un tale poeta. Non ne tenta traghettamenti prosodici, piuttosto mira a conservarne la sintassi fratta, la tensione della ricerca attorno a "come lievita la poesia". Intatte le faglie temporali del pensiero "interno" che muove verso la parola non ancora strutturata - "Ho dimenticato la parola che volevo dire". Una parola incipiente, che ancora tenta la sortita, "mugolio / di sonore conchiglie entro la sabbia". 